\title{
Persistent infection of Mycobacterium tuberculosis in a patient with Behçet's disease after rotator cuff repair surgery: A case report
}

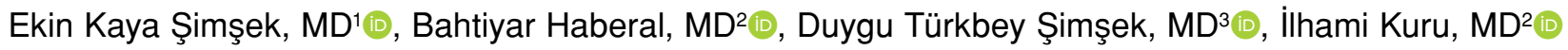 \\ ${ }^{1}$ Department of Orthopedics and Traumatology, Kaçkar State Hospital, Rize, Turkey \\ ${ }^{2}$ Department of Orthopedics and Traumatology, Başkent University Ankara Hospital, Ankara, Turkey \\ ${ }^{3}$ Department of Pathology, Başkent University Ankara Hospital, Ankara, Turkey
}

Tuberculosis (TB) infection is still a common health problem in low socioeconomic populations in developing countries around the world. ${ }^{[1]}$ Osteoarticular TB is a disease that rarely occurs outside of the vertebral column and is of ten diagnosed late or misdiagnosed due to its atypical presentation, particularly in non-weight bearing joints. Shoulder TB accounts for about 1 to $2 \%$ of extrapulmonary TB cases. ${ }^{[2,3]}$ Although osteoarticular TB can develope secondary to pulmonary $\mathrm{TB}$, it has recently been proven to occur without pulmonary and systemic symptoms in the majority of patients..$^{[4,5]}$ As a subsequent result, its diagnosis is often problematic, and the delay in a diagnosis can lead to destructive ramifications for joints and periarticular tissues. In this particular case, we present a case of Behçet's

Received: January 26, 2021

Accepted: March 28, 2021

Published online: November 19, 2021

Correspondence: Bahtiyar Haberal, MD. Başkent Üniversitesi Tıp Fakültesi, Ortopedi ve Travmatoloji Anabilim Dalı,

06490 Bahçelievler, Ankara, Türkiye.

E-mail: bahtiyarhaberal@hotmail.com

Doi: $10.52312 /$ jdrs.2021.37

Citation: Kaya Simșek E, Haberal B, Türkbey Simşek D, Kuru İ. Persistent infection of Mycobacterium tuberculosis in a patient with Behçet's disease after rotator cuff repair surgery: A case report. Jt Dis Relat Surg 2021;32(3):786-791.

(92021 All right reserved by the Turkish Joint Diseases Foundation

This is an open access article under the terms of the Creative Commons Attribution-NonCommercial License, which permits use, distribution and reproduction in any medium, provided the original work is properly cited and is not used for commercial purposes (http://creativecommons.org/licenses/by-nc/4.0/).

\section{ABSTRACT}

Tuberculosis (TB) infection is a common health problem in low socioeconomic populations in developing countries. Osteoarticular TB is a disease that rarely occurs outside the vertebral column and is often diagnosed late or misdiagnosed, particularly in non-weight bearing joints. A 51-year-old female patient with Behçet's disease presented with shoulder TB following rotator cuff repair surgery, leading to joint resection as a result of recurrent infections with no reproduction in culture samples due to delayed diagnosis. Surgical excision was proposed for the treatment of draining a mass with a sinus tract opening into the joint on the anterior aspect of the right shoulder. A diagnosis was able to be reached after 13 operations, based on the presence of TB bacilli in the pathological examination of the samples. In conclusion, TB should be considered in the absence of reproduction in culture samples taken during persistent post-surgical infections.

Keywords: Mycobacterium tuberculosis, shoulder, rotator cuff, surgery.

syndrome in whom the definitive diagnosis of shoulder TB was able to be made after 13 operations over a four-year period.

\section{CASE REPORT}

A 51-year-old patient was admitted to the outpatient department with swelling to the right shoulder and surrounding area, a skin wound, restriction in shoulder movement, and pain. The patient received rotator cuff repair surgery four years prior at an external clinic. Following the initial repair, she was operated on a total of 13 more times to address the infection, based on persistent pain and wound discharge, finally resulting in a final bone resection. The patient reported that the erythema, swelling, and tenderness in her right shoulder reappeared one 


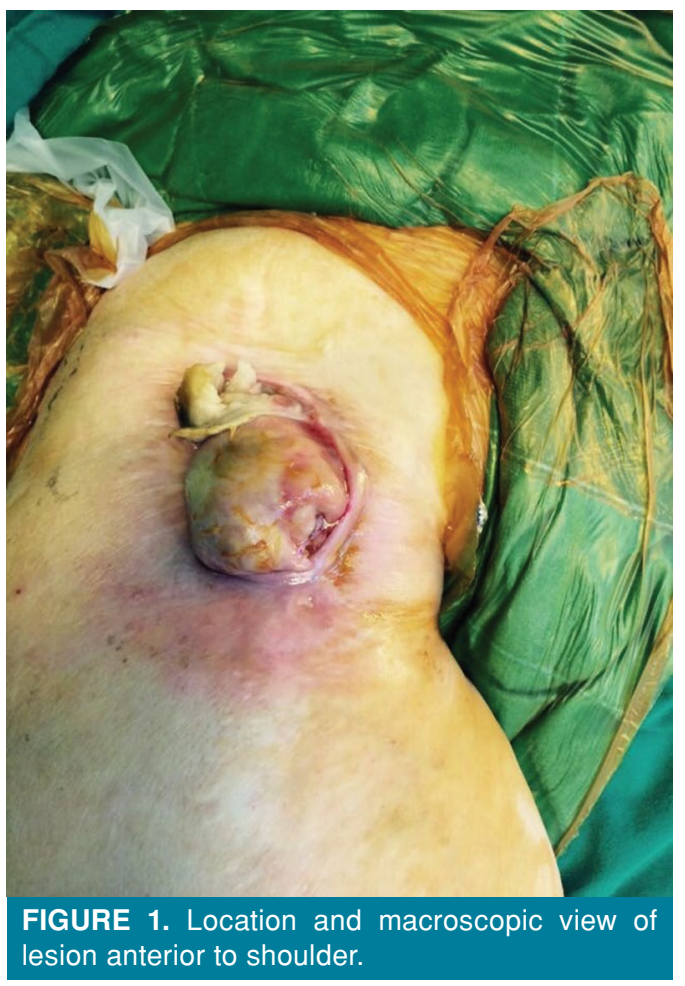

month before admission to this current clinic. Her medical history revealed that she was under follow-up by the rheumatology department at another hospital for Behçet's disease and that she did not receive antitumor necrosis factor (anti-TNF) drug therapy at the time of admission; however, she received oral steroids for a significant period of time. The patient was also being treated for hypertension.

A physical examination revealed a yellow-brown swollen nodular lesion with a necrotic surface and lobular contour, located on the anterior part of the shoulder joint, approximately $7.5 \mathrm{~cm}$ in size (Figure 1). The circumference of the shoulder was oedematous, and shoulder movements were painful and limited in all directions. There were no palpable lymph nodes around the shoulder. During radiographic examination, contrast-enhanced magnetic resonance imaging (MRI) was performed, following the inspection of previous direct radiographs provided by the patient herself. Contrast-enhanced MRI assessment revealed a complete resorption appearance in the right humeral head, extending to proximal diaphysis; soft tissue appearances in the resorption zone, which were thought to belong to severe hypertrophic synovial tissues; full-thickness defect in the subcutaneous adipose tissue at shoulder level and in the deltoid muscle, extending to humeral head level; and condensed liquid collection and continuity of this collection within the proximal resorbed humerus zone. The supraspinatus, infraspinatus, and subscapularis muscles all presented with a highly fatty atrophy appearance. However, MRI was inadequate to clearly identify whether the fluids were infected (Figure 2).

In her routine blood tests, leukocyte was $8.2 \mathrm{Tu} / \mathrm{L}$, erythrocyte sedimentation rate was $77 \mathrm{~mm} / \mathrm{h}$, and C-reactive protein was $19.14 \mathrm{mg} / \mathrm{L}$. A culture sample was taken from the wound discharge on the patient's shoulder; however, it showed no reproduction during culture sample monitoring. After clinical evaluation, septic arthritis was considered. To make a microbiological diagnosis and to obtain pathology and culture samples for differential diagnosis of other possible pathologies, such as neoplasms, an excision of the sinus tract, extending into the joint, and skin lesion and joint debridement were planned. A written informed consent was obtained from the patient.
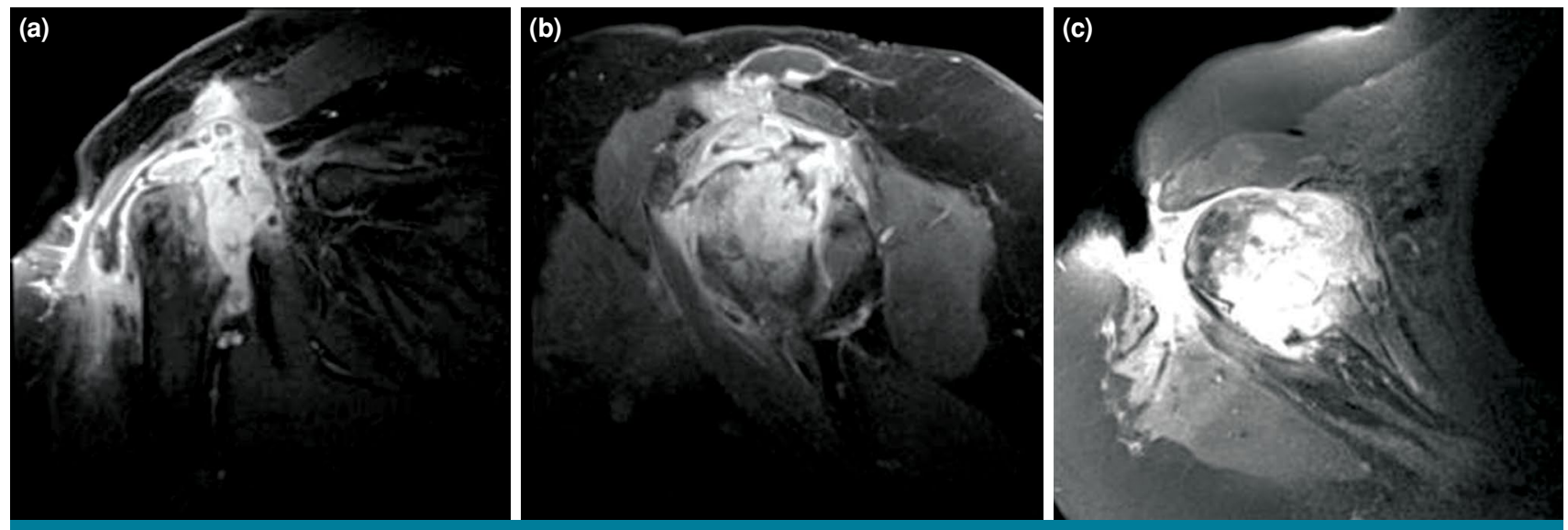

FIGURE 2. Preoperative T1-weighted images of affected shoulder. (a) Coronal view. (b) Sagittal view. (c) Axial view. 
The patient was operated on under general anesthesia. To avoid affecting the culture samples taken during surgery, intravenous cefazolin sodium (1 g) (Cezol; Deva Holding AS, Istanbul, Turkey) was administered for surgical prophylaxis, after the samples were taken. During surgery, the patient was in a beach chair position with an arm board to support her right elbow and forearm. Prior to surgery, a Z-plasty incision was planned to allow for closure of the skin after the mass was removed. Following the incision around the mass, dissection was carried out and advanced through the deltopectoral space. Preserving the axillary vessel-nerve package, the mass, including the joint capsule, was excised extensively along with the anterior fibers of the deltoid muscle. Subsequently, the shoulder joint was explored. Dead bone residues and infected synovial tissues were debrided, until bleeding was observed. At this stage, culture samples were taken for microbiological examination, followed by irrigation of the surgical field with approximately six liters of saline solution. A suction drain was inserted after bleeding was under control, and the wound was closed. Following surgery, the patient's shoulder was dressed using a Velpeau bandage. Physical therapy for the patient's elbow and wrist commenced on the first postoperative day. The suction drain was removed on the third postoperative day, and the patient was discharged. The remaining bone stock after surgery was evaluated on postoperative Day 15 by direct radiography (Figure 3). Macroscopic

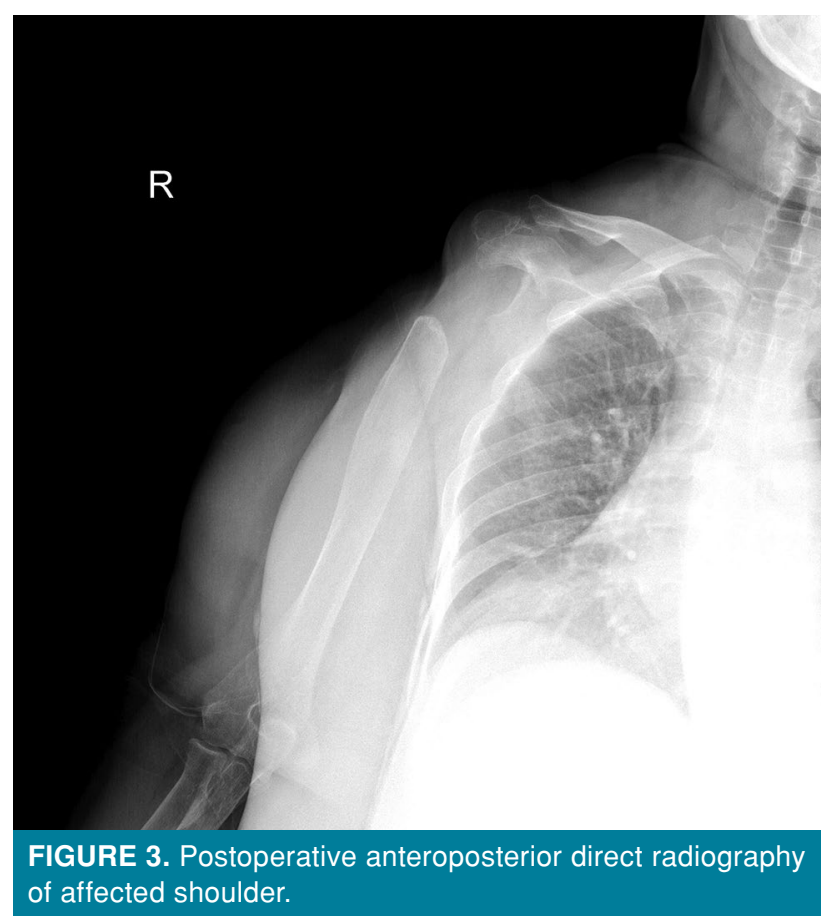

pathological examination revealed a $14.5 \times 5.8 \times 3.5-\mathrm{cm}$ mass with papillary infoldings. On the cut surface, irregularly delineated white confluent foci were visible (Figure 4). Microscopically, granulomas with Langhans giant cells and caseous necrosis could be seen. Some granulomas were cuffed by lymphocytes (Figures 5 and 6). Acid-fast bacillus (AFB) stain showed TB bacilli in the necrotic areas and histiocytes (Figure 7); however, there was no reproduction in the joint fluid culture. The TB treatment was initiated by consulting with the Department of Infectious Diseases and Clinical Microbiology. At six months after surgery, TB treatment using a combination of drugs was ongoing, while the patient no longer experienced symptoms and pain related to the infection. Direct radiographic evaluation of the shoulder joint was made (Figure 8 ), and joint reconstruction was planned for the end of the medication treatment.
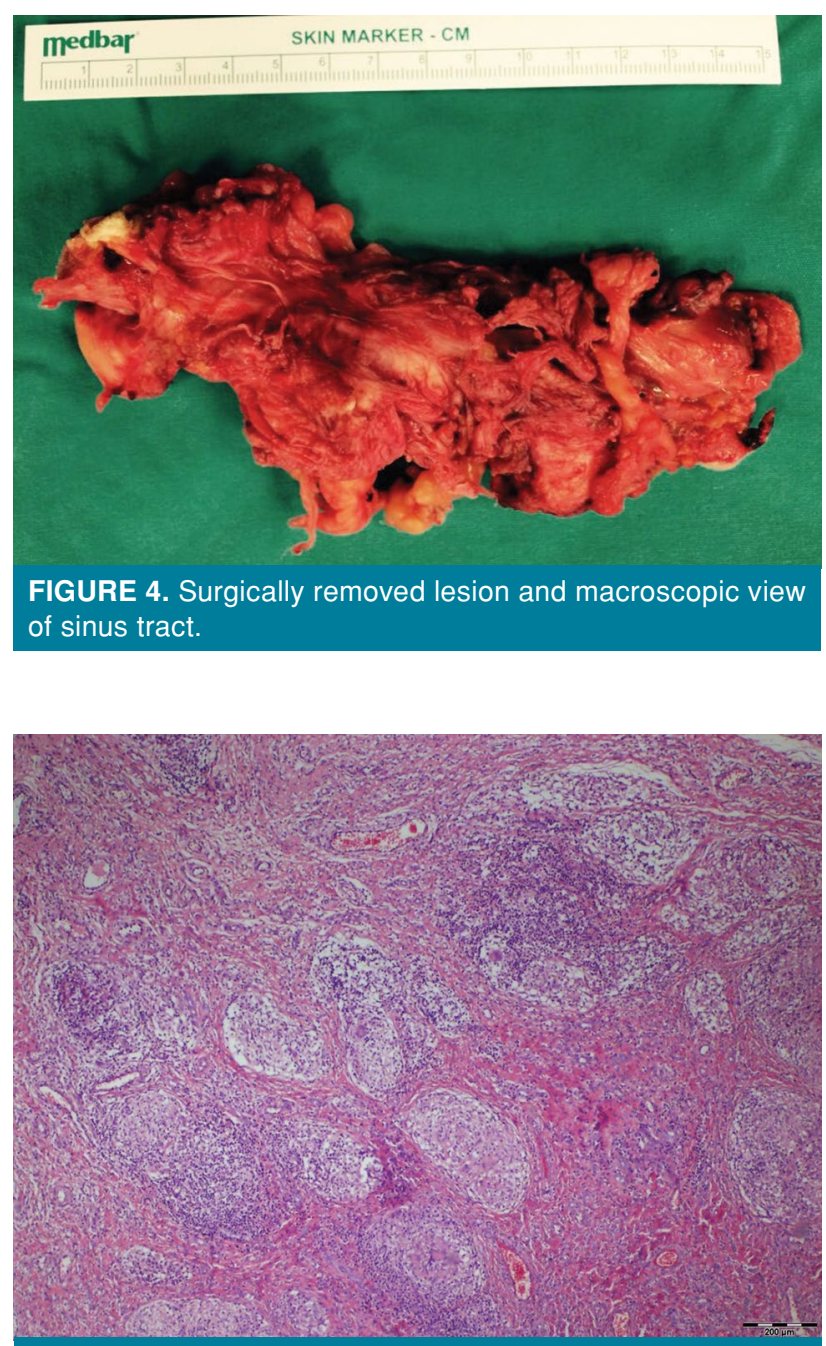

FIGURE 5. Granulomas composed of epithelioid cells, a few giant cells, and lymphocyte cuffs $(\mathrm{H}-\mathrm{E}, \times 40)$. 


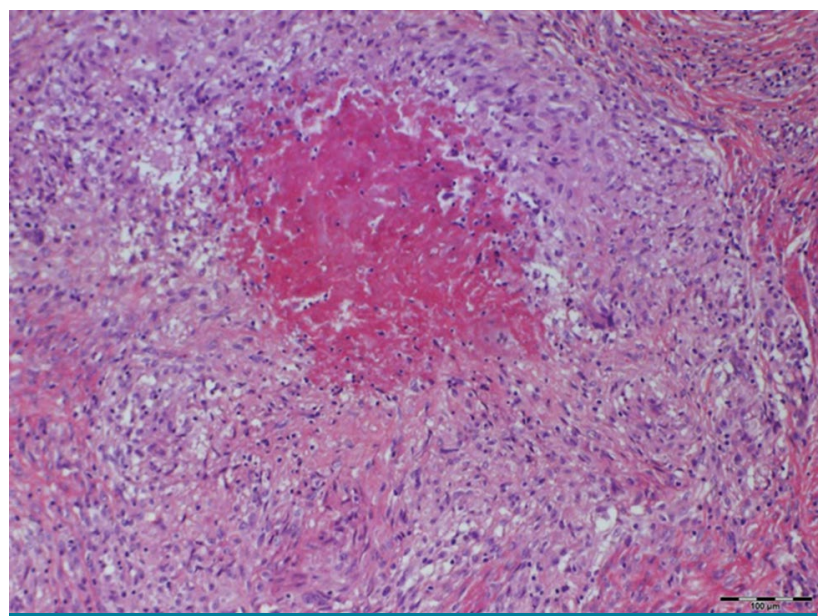

FIGURE 6. A large caseating granuloma with palisading epithelioid cells $(H-E, \times 100)$.

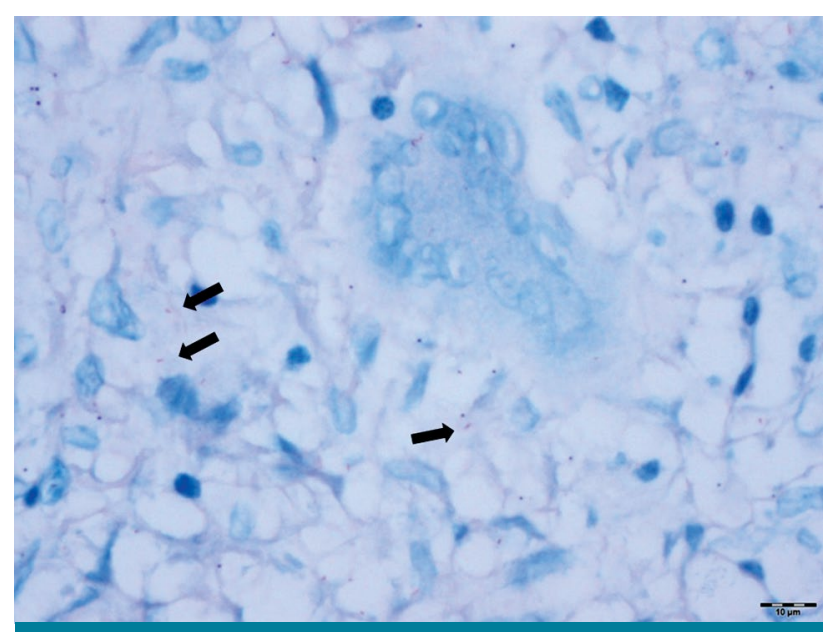

FIGURE 7. Acid-fast bacillus staining showing tuberculosis bacilli (black arrows) in giant cells and histiocytes $(\times 600)$.

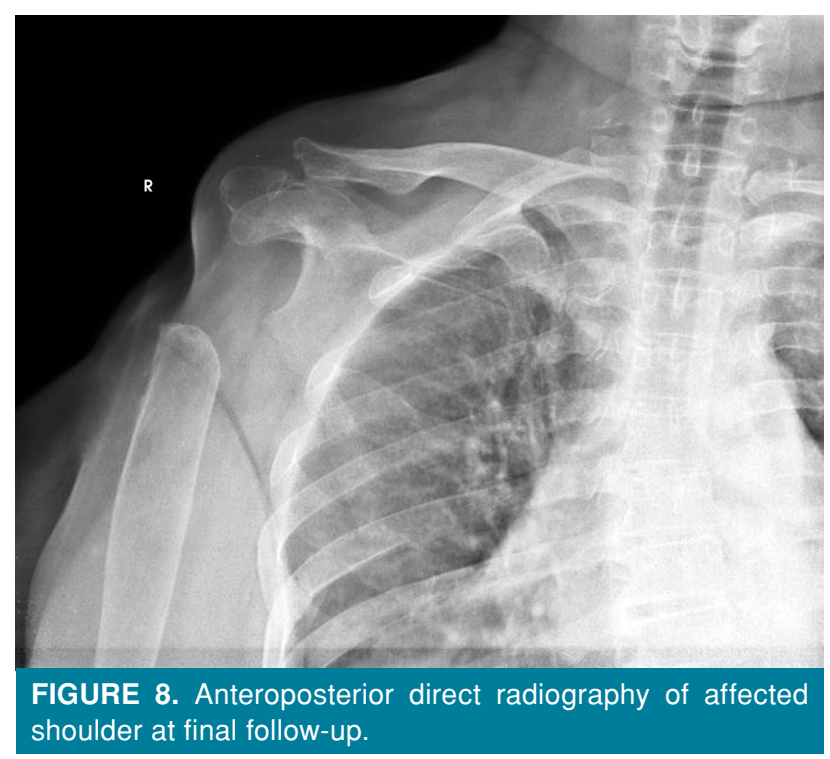

\section{DISCUSSION}

This article presents a case of shoulder TB in a female adult with Behçet's disease following rotator cuff repair surgery, leading to joint resection as a result of recurrent infection, due to delayed microbiological diagnosis. Cases of extrapulmonary TB, which are rare in non-weight bearing joints and, thus, problematic to diagnose early, have a devastating effect on joints and periarticular tissues. Early diagnosis is only possible with a detailed medical history of the patient and high level of clinical suspicion. This case report aims to contribute to existing literature, by drawing attention to extrapulmonary TB cases that initially go undiagnosed and cause significant and unexpected issues for diagnosis in daily clinical practice.

Extrapulmonary TB accounts for $15 \%$ of TB cases and affects the lymphatic system, genitourinary system, and musculoskeletal system. It is particularly common in patients with human immunodeficiency virus, rheumatic diseases, immunosuppression due to organ transplantation, and in elderly patients. ${ }^{[1]}$ In cases involving the musculoskeletal system, the most common outcome is spondylitis, due to the involvement of the spine; the second most common outcome is arthritis, due to the involvement of large joints. Tuberculosis in large weight-bearing joints, such as the hips and knees, is common, but shoulder TB is quite rare..$^{[6]}$ The infection proceeds incognito and causes similar symptoms to degenerative diseases of the shoulder joint. Patients with shoulder TB often receive treatment for diagnoses such as adhesive capsulitis, a rotator cuff tear, and arthritis of the shoulder joint and, therefore, correct diagnosis is delayed..$^{[1,7]}$ In this particular case, the patient was first diagnosed with a rotator cuff rupture, leading to an operation and delayed diagnosis of the underlying issue.

Behçet's disease is a multisystemic chronic inflammatory vasculitis with an unknown etiology and pathogenesis. It is frequently observed in patients along the Silk Road in Asia and Central Asia. ${ }^{[8]}$ Mycobacterium tuberculosis is known to trigger Behçet's disease. ${ }^{[9]}$ Moreover, the incidence of TB infection in Behçet's disease sufferers increases significantly, due to both cell-mediated immune system disorders observed in these patients, and the medications used in the treatment of this disease that suppress the immune system, in particular, antiTNF agents. ${ }^{[8]}$ Consequently, TB infection in Behçet's disease sufferers can be both the cause and the result. In the present case, however, the patient was not using anti-TNF agents and TB infection developed. Detailed questioning concerning the patient's history 
to reveal possible underlying causes associated with their complaint and an examination of the persistent wound infection facilitate a diagnosis by providing the correct approach to solving the problem.

There are three types of shoulder TB: Wet (caries exudata), dry (caries sicca), and mobile (caries mobile). In the dry type, which is common in adults, joint movements are painful and limited, while in the wet type, which is more common in children, cold abscesses and swelling around the shoulder are often apparent. ${ }^{[10]}$ In the mobile type of shoulder $\mathrm{TB}$, the active range of motion (ROM) is limited, but the passive ROM is open. Subcutaneous tissue adjacent to a joint and the formation of abscesses and fistulas on the skin is typical for TB arthritis. ${ }^{[1]}$ Despite being uncommon in adults, this particular case is a wet type shoulder TB, confirmed by the presentation of wide cold abscesses. The ulcerative lesion at the front of the patient's shoulder and fistula extending to the joint are typical indicators of shoulder TB.

In infections where the joint is involved, the first visualization is done with a direct roentgenogram. Typical direct roentgenogram findings of TB arthritis are juxta-articular osteoporosis, subchondral erosions, and stenosis in the joint spacing. These three findings are referred to as Phemister's triad. ${ }^{[11]}$ In later stages, it presents as severe joint destruction. ${ }^{[10,12]}$ The presence of sclerosis around the lytic sites indicates a chronic infection. ${ }^{[1]}$ Computed tomography and ultrasonography can be also used in the diagnosis of TB arthritis; however, MRI is the preferred method, as it has the ability to show soft tissue involvement and helps in the differential diagnosis. ${ }^{[13]}$ Its ability to show periarticular abscesses and sinus tracts, particularly in the later stages of infection, makes surgical planning easier. ${ }^{[14]}$ In this case, intra-articular fluid collection and sinus tracts were clearly evaluated by MRI and complete excision with hypertrophic synovial tissue was achieved.

Treatment of shoulder TB includes multi-drug anti-TB treatment, repetitive debridement, irrigation, and immobilization. Restriction of movement in the shoulder joint after surgery is the most commonly reported issue. ${ }^{[10]}$ Arthrodesis and resection arthroplasty are indicated in late diagnosed cases. Although these practices relieve the associated pain, they result in poor functional results. ${ }^{[3]}$ Even if shoulder movement is retained following resection arthroplasty, the ROM would be limited, due to the loss of the shoulder fulcrum. In a case report by Luenam and Kosiyatrakul, ${ }^{[3]}$ one-stage cemented shoulder hemiarthroplasty was used to treat a 50-year-old male patient, whose symptoms were ongoing for five years, and a 42-year-old male patient who suffered from symptoms for three years. They reported acceptable levels of patient satisfaction, despite some loss of shoulder function in the five-year-long case, during monitoring after the 12-month multi-drug anti-TB treatment. In this case, complete excision of infected tissues was achieved to shed light on the pathology causing the infection. Following the completion of the drug treatment for rotator cuff loss, glenoid joint face loss, and bone stock deficiency, resulting from numerous surgical debridement, a three-dimensional reconstructed individual shoulder arthroplasty was planned. However, in the second year after surgery, the patient died as a result of complications from ovarian cancer.

Osteoarticular TB can mimic acute pyogenic arthritis and osteogenic sarcoma. However, in the differential diagnosis eosinophilic granuloma, chondroblastoma, clear cell chondrosarcoma, sarcoidosis, brucellosis, fungal infections, rheumatoid arthritis, metastasis, pigmented villonodular synovitis, synovial chondromatosis, and pyogenic osteomyelitis should be also considered. Pyogenic (bacterial) arthritis, the most common in differential diagnosis, affects a single joint, but has a more acute onset. It can develop from trauma and bacteremia. ${ }^{[5,10]}$ Therefore, microbiological and histopathological examination becomes crucial for the diagnosis. The Ziehl-Neelsen staining and/or positive results in cultures taken from the affected tissue are considered the gold standard in the diagnosis of osteoarticular $\mathrm{TB}^{\left[{ }^{[2]}\right.}$ In these cases, the diagnosis rates of AFB staining and conventional culture are low. ${ }^{[10]}$ Moreover, microbiological diagnosis becomes increasingly difficult due to numerous operations that the patient undergo, recurrent infections, and long-term antibiotic use, prior to their admission to the clinic. In this case, histopathological examination provided the greatest contribution to diagnosis, while the culture results were negative. As is also present in this particular case, a histopathological evaluation of TB infection shows a granulomatous reaction accompanied by caseous necrosis.

In conclusion, in the light of this report, TB should be considered, when reproduction in culture samples taken in the event of persistent infections developed after surgery is absent. Detailing of the patient's clinical history, determination of predisposing comorbidities implicated in the development of infection, and a histopathological evaluation, combined with considering the possibility of a TB 
infection, would shorten the time to diagnosis. Timely diagnosis and treatment would ensure the protection of the affected joint and its functions.

\section{Declaration of conflicting interests}

The authors declared no conflicts of interest with respect to the authorship and/or publication of this article.

\section{Funding}

The authors received no financial support for the research and/or authorship of this article.

\section{REFERENCES}

1. Kizildag B, Sener A, Komurcu E, Karatag O, Kosar S. Glenohumeral joint tuberculosis with multiple cold abscesses: An uncommon cause of shoulder pain. BMJ Case Rep 2013;2013:bcr2013200592.

2. Deshmukh A, Deo S, Salgia AK, Agarwal T. A Rare unusual case presentation of the tuberculosis of the shoulder joint. J Orthop Case Rep 2013;3:23-5.

3. Luenam S, Kosiyatrakul A. Immediate cementless hemiarthroplasty for severe destructive glenohumeral tuberculous arthritis. Case Rep Orthop 2013;2013:426102.

4. Birole U, Ranade A, Mone M. A case report of an unusual case of tuberculous osteomyelitis causing spontaneous pathological fracture of humerus in a middle aged female. J Orthop Case Rep 2017;7:41-5.
5. Cheng J, Feng S, Lei H, Huo W, Feng H. Tuberculosis of acromioclavicular joint: A case report. BMC Infect Dis 2019;19:111.

6. Dev R. Tuberculosis of shoulder joint an unusual case presentation. Int J Anat Radiol Surg 2018;7:RC04-RC06.

7. Li JQ, Tang KL, Xu HT, Li QY, Zhang SX. Glenohumeral joint tuberculosis that mimics frozen shoulder: A retrospective analysis. J Shoulder Elbow Surg 2012;21:1207-12.

8. Liu Y, Zhang L, Zhou Z, Sun L, Zhou B, Liu X, et al. Clinical features and risk factors of active tuberculosis in patients with Behçet's disease. J Immunol Res 2020;2020:2528676.

9. Freitas SM, Marques JS, Grilo A, Gomes R, Gonçalves FM. Behçet's disease and tuberculosis: A complex relationship. Eur J Case Rep Intern Med 2020;7:001354.

10. Agarwal A, Kumar A, Shaharyar A, Bhat MS. Shoulder tuberculosis in children: A report of two cases. J Orthop Surg (Hong Kong) 2015;23:398-401.

11. Goel V, Sharma PK, Jain J, Yadav U, Devgan A, Bhardwaj N. Caries sicca: Tuberculosis of glenohumeral joint. Int J Res Orthop 2019;5:747-9.

12. Aggarwal R, Roy K, Nemade P, Purohit S. XDR-TB of the shoulder presenting as a lytic lesion in the proximal humerus. Int J Tuberc Lung Dis 2016;20:283-4.

13. Leonard MK, Blumberg HM. Musculoskeletal tuberculosis. Microbiol Spectr 2017;5:1-21.

14. Atik OŞ. Is there something new and interesting in my article? Eklem Hastalik Cerrahisi 2019;30:69. 